Voix et Images

voixetimages

\title{
La théâtralité fragmentée
}

Lucie Robert

Volume 20, numéro 3 (60), printemps 1995

André Brochu

URI : https://id.erudit.org/iderudit/201203ar

DOI : https://doi.org/10.7202/201203ar

Aller au sommaire du numéro

Éditeur(s)

Université du Québec à Montréal

\section{ISSN}

0318-9201 (imprimé)

1705-933X (numérique)

Découvrir la revue

Citer cet article

Robert, L. (1995). La théâtralité fragmentée. Voix et Images, 20(3), 721-730.

https://doi.org/10.7202/201203ar d'utilisation que vous pouvez consulter en ligne.

https://apropos.erudit.org/fr/usagers/politique-dutilisation/ 


\section{Dramaturgie}

\section{La théâtralité fragmentée}

\section{Lucie Robert, Université du Québec à Montréal}

Il reste peu de traces du travail, pourtant essentiel, que conduit Jean-Pierre Ronfard au Nouveau Théâtre expérimental de Montréal. Aussi doit-on souligner la parution des Cinq Études ${ }^{1}$ comme un heureux événement, comme une clé d'accès à une théâtralité poussée toujours plus loin jusqu'à en redéfinir les frontières. Le titre même du livre décrit la nature du travail, le mot "étude "renvoyant explicitement à l'œuvre musicale de Frédéric Chopin, mais on pourrait tout aussi bien l'envisager dans son rapport aux beaux-arts, c'est-à-dire au travail en procès, essai ou exercice sur une théâtralité d'abord découpée en fragmë̀nts constitutifs puis explorée dans "une réflexion critique portant sur l'un ou l'autre des éléments de l'art théâtral (p. 7). Qu'estce que ceci? Qu'est-ce que cela? Est-il possible de ...? demande l'auteur constamment. La réponse à ces questions se trouve inscrite dans un nouveau spectacle qui, tout en offrant le caractère achevé nécessaire à la représentation, conserve néanmoins la trace de la démarche suivie. Les Cinq Études ne sont donc pas des pièces de théâtre comme telles, ni même des textes dramatiques, mais bien des objets hybrides, des "objets dramatiques", écrivait Claude Gauvreau. Il en résulte que chaque texte emprunte largement le parcours de la narration et de l'argumentation, l'un et l'autre étrangers à une dramaturgie véritable, et que l'anecdote, généralement simple et conventionnelle, est voulue ainsi pour faciliter le travail exploratoire. En vain cherchera-t-on ici une réflexion sur la vie et sur le monde: les Cinq Études ne soulèvent de poussière que celle d'une théâtralité constamment redéfinie.

Les cinq textes publiés ici ont tous été créés au Nouveau Théâtre expérimental de Montréal entre 1986 et 1993 . La première étude, "Les objets parlent", montre le caractère immédiatement social, icônique, signifiant de l'objet, qu'il soit accessoire, décor ou costume. Ces objets sont seuls en scène: aucun comédien ne les manipule. De sorte que le texte présenté est une longue didascalie en dix tableaux. L'on perçoit toutefois clairement que les objets ici sont les personnages réels, que, seuls, ils sont néanmoins porteurs d'une série d'anecdotes simples, que leur déplacement et leúr transformation créent des effets de temporalité: le mouvement des objets intimes, dans une salle de 
bains, laisse ainsi entrevoir l'aventure amoureuse qui se déploie ailleurs. Poussant la logique de l'objet à son extrême limite, Ronfard va jusqu'à transformer les spectateurs en objets alors que ce sont les portemanteaux, où les spectateurs réels ont déposé leurs effets, qui se mettent en mouvement, devenant en quelque sorte l'icône de la situation de représentation. Certains traits d'avant-garde rappellent la dramaturgie d'Eugène Ionesco, tel ce réfrigérateur fou qui livre une marchandise incongrue. En revanche, cet autre texte qu'est "Corps en mouvement " est plus difficile à lire puisque, par définition, le mouvement et le corps fuient constamment les usages linguistiques. La superposition des divers systèmes de signes, les arts martiaux, le langage corporel sexué, le corps amoureux rendent la difficulté encore plus grande. De sorte que, si l'on saisit bien le projet mis en œuvre, on en voit mal la réalisation réelle.

À ces deux réflexions sur l'espace et le jeu, s'ajoutent trois réflexions qui touchent plus étroitement le langage et la dramaturgie. "Autour de Phèdre", essai sur le tragique, et "La Voix d'Orphée ", réflexion sur la voix, sont construites de la même manière: le metteur en scène agit comme meneur de jeu et comme voix narrative, les comédiens sont là pour donner forme aux rôles. Les deux études fondent la réflexion en retrouvant le mythe porteur de la plus grande signification et empruntent plusieurs passages à la tradition grecque, au théâtre de Racine ou à des œuvres musicales emblématiques. Dans ces deux cas, les plus satisfaisants de l'ensemble, nous sommes déjà dans l'ordre d'un nouveau texte, presque une dramaturgie en soi. "Violoncelle et voix", la dernière étude, explore la construction du dialogue. Son intérêt réside dans l'établissement de deux voix distinctes: la première utilise le langage courant (c'est-à-dire que, conformément aux usages courants du dialogue, cette voix énonce des répliques à contenu linguistique), l'autre est musicale (le violoncelle doit ainsi répondre à la première voix). Le résultat permet de saisir l'essence même du dialogue qui est un échange avant d'être une série de messages. Le texte, tel qu'il est publié, repose cependant sur une fiction: faute de pouvoir faire entendre la musique, Ronfard a recomposé le texte (linguistique) que doit faire entendre le violoncelle. Aussi, à la lecture, n'est-on guère en mesure de saisir tout l'intérêt du projet.

Le travail expérimental de Ronfard, tel qu'il nous parvient dans les Cinq Études, possède un certain nombre d'éléments caractéristiques: la théâtralité y est décomposée en éléments distincts (l'objet, le corps, la voix, le dialogue, le tragique) et chacun est traité en profondeur par le recours à des anecdotes simples (généralement le rapport 
amoureux, hyper-codé dans une société comme la nôtre) et à des langages pré-construits: la mythologie grecque (Ronfard est helléniste de formation), les arts martiaux, la musique, le Phèdre de Racine, etc. Ce qui retient l'attention est le caractère classique de ces systèmes référentiels : classiques en ce qu'ils montrent un rapport étroit aux traditions de la littérature et du théâtre occidental; classiques également en ce qu'ils sont largement connus du public auquel Ronfard s'adresse. De sorte que l'on peut croire que le deuxième élément est celui qui permet au spectateur de saisir la nature et l'orientation du travail d'expérimentation alors que le premier est ce qui lui donne sa profondeur. Il s'établit ainsi une dialectique de la tradition et de la nouveauté, qui caractérise tout aussi bien le travail plus conventionnel du metteur en scène et dramaturge et qui, de l'avis de Ronfard luimême, manque généralement au théâtre québécois actuel ${ }^{2}$.

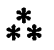

"Autour de Phèdre" aurait pu servir de source à Chantale Boileau dans sa réflexion sur le tragique. Dans Les Formes du tragique. Essai suivi de La Mort de Blanche. Théâtre ${ }^{3}$, d'abord déposé comme un mémoire de maîtrise en études théâtrales à l'Université du Québec à Montréal, l'usage traditionnel de recourir en premier lieu aux sources secondes, c'est-à-dire aux écrits des philosophes, critiques et historiens, a cependant prévalu. Aucun compte n'est véritablement tenu des réflexions des dramaturges eux-mêmes; peu de renvois sont faits au travail des écrivains. Et pourtant, l'objectif premier de l'auteure est la pièce elle-même, cette Mort de Blanche, tragique, dramatique et mélodramatique à la fois, bien qu'à des degrés divers. Je crois toujours que les écrivains tirent un plus grand profit à se confronter à l'écriture des autres qu'à envisager, trop rapidement, celle des analystes. L'écriture m'apparaît comme un constant mouvement d'imitation et de distanciation, de sorte que l'identité du genre prime toujours. Et si les jeunes critiques ont un intérêt immédiat à lire et relire les critiques qui les précèdént, il me semble que les dramaturges devraient d'abord relire la dramaturgie.

En conséquence, la réflexion de Boileau sur Les Formes du tragique reste tout en surface, prenant appui sur quelques écrits théoriques déjà anciens, laissant une part un peu maigre à mon avis aux classiques (Aristote, Corneille, Racine) plutôt lus à travers des études de seconde main. Le titre de l'essai est quelque peu trompeur, puisque la tragédie comme telle n'occupe que le tiers de l'essai, les deux 
autres parties portant sur le drame et sur le mélodrame, bien que ces trois genres soient ici conçus comme autant de formes du tragique, lui-même réduit à "la reconduction sur scène de la chute de l'être humain" (p. 17). Cette définition est radicalement différente de celle de Ronfard qui voyait surtout le tragique dans la manipulation, la soumission de la destinée humaine aux caprices des dieux.

C'est donc "la chute de l'être humain" que l'auteure tente de mettre en scène dans sa propre pièce, La Mort de Blanche, créée en octobre 1992 au Studio d'essai Claude-Gauvreau de l'Université du Québec à Montréal. Sortie du contexte un peu prétentieux auquel la contraint l'essai, la pièce, toutefois, n'est pas sans intérêt, bien que l'écriture se trouve coincée par le cadre générique rigide établi dans la réflexion théorique. À la lecture, je n'ai pas pu m'empêcher de penser à Tennessee Williams, à ces personnages porteurs d'une tare originelle, dont ils ignorent parfois jusqu'à l'existence, mais qui n'en détermine pas moins, hors de toute volonté, leurs comportements. L'action construite par Chantale Boileau est provoquée par la mort de Blanche, précisément, à qui sa fille Johanne vient rendre un dernier hommage. La mère et la fille se détestent et ne se sont jamais revues depuis le jour où, vingt-quatre ans plus tôt, Johanne a quitté la maison pour la Grèce où elle a fait carrière comme chanteuse. L'heure de la mort sera donc celui de régler des comptes entre une mère, qui souffre de psoriasis, déteste sa condition de femme, d'épouse et de mère (elle a eu sept enfants), et sa fille, qui aurait souhaité plus d'amour, de dignité et d'encouragement.

La pièce procède selon un des canons actuels les plus prégnants de l'écriture dramaturgique: celle de la fracture du personnage. La fracture opère ici dans le temps (les personnages oscillent entre le présent et le passé, remontant le temps jusqu'à l'origine) et dans le sujet lui-même, puisque nous avons, sur scène, deux Blanche, l'une acariâtre, l'autre sereine. Le bourreau n'est pas celle qu'on croit: la révélation du secret de Johanne résout la fracture identitaire et permet à Blanche de reposer, enfin, en paix. Mais de tragique, pas vraiment. Nous sommes essentiellement dans l'ordre du drame classique, écrit dans une rhétorique toute postmoderne, dont l'effet commence malheureusement à s'user.

Créée le 15 mars 1994 à la salle Denise-Pelletier de la Nouvelle Compagnie théâtrale, dans une production de Ma chère Pauline et une 
mise en scène de Sylvain Hétu, L'Ombre de to $i^{4}$, de Sylvie Provost, présente deux personnages, Julie et Jean-Philippe. Julie vient de demander le divorce et Jean-Philippe fait irruption dans l'appartement où elle s'est réfugiée pour exiger, sous la menace d'un revolver, des explications. À ces deux personnages, s'ajoutent des voix qui arrivent sur scène à travers divers médias: le téléphone, la télévision, l'intercom. De sorte que les deux personnages demeurent seuls dans un espace clos tout en étant perméables à ce qui vient d'ailleurs. Le procédé permet de concentrer l'action et le dialogue autour du couple et de situer le conflit dramatique au plan discursif. Hélène Pedneault, parmi d'autres, avait déjà utilisé cette technique dans La Déposition.

Techniquement, et comme la précédente, la pièce est structurée par une remontée dans le temps. Nous retrouvons ici la rhétorique du personnage fragmenté, puisque les deux protagonistes passent constamment d'un temps à l'autre, remontant le fil de leur histoire depuis leur première rencontre jusqu'à la séparation. L'action se déroule ainsi sur dix ans et le point de départ, précise la didascalie initiale, doit se situer dans le présent du spectateur. De sorte que l'action comme telle se passe en l'an 2004 (la date doit être modifiée à chaque nouvelle production) et que, à l'histoire somme toute banale d'un divorce se greffe une réflexion sur l'avenir de notre présent. Outre un jeu technologique sur les réalités virtuelles, on ne voit cependant pas bien ce qu'une telle perspective apporte au drame, celui-ci étant par trop conventionnel. Le dialogue reste un combat de mots où les deux actants revivent, en l'analysant, leur rapport amoureux, cherchant qui, de l'un ou de l'autre, est davantage responsable de l'échec. La dynamique de l'ensemble repose sur l'inversion des rôles attendus: le bourreau se décompose en révélant sa faiblesse réelle alors que la victime développe au contraire lá force propre qui l'a conduite à demander le divorce.

\section{**}

Comme pratiquement toutes les pièces qui nous proviennent du Nouvel Ontario, $E d d y^{5}$ problématise la situation des francophones hors Québec, en mettant en scène des personnages qui, ayant quitté les mines de Sudbury, se trouvent coupés de leurs racines. La dramaturgie franco-ontarienne actuelle se trouve ainsi à réintroduire, dans la littérature, une réflexion sur l'aliénation, dont l'intérêt est néanmoins maintenu par l'ambivalence de la situation des personnages. En quittant Sudbury et ses mines, ceux-ci atteignent généralement un 
certain succès. Comment qualifier autrement le fait d'échapper aux dangers de la mine (même pour un petit snack-bar montréalais), à la pauvreté et à la violence paternelle, constante dans la dramaturgie du Nouvel Ontario, chez Dalpé en particulier, mais aussi chez Michel Ouellette, notamment dans French Town? Le succès est néanmoins mitigé puisqu'il est obtenu par le renoncement à la tradition et à la famille, fondatrices de l'identité.

Les rêves sont de toute nature et celui d'Eddy était de devenir un grand boxeur. C'est son frère Jacques, mineur comme son père, qui lui aura fourni les moyens de partir et de lancer à Montréal sa nouvelle carrière. Eddy a eu son heure de gloire, ayant remporté plusieurs combats, avant de se retrouver propriétaire d'un snack-bar qui lui assure de maigres revenus et lui permet d'entretenir l'illusion d'être resté un grand entraîneur-gérant, ce que lui conteste le fonctionnaire de l'impôt, qui ne voit chez lui qu'un vague amateur. Mais voilà que Vic, le fils de Jacques, reprend le rêve de son oncle. La pièce s'engage ainsi sur l'arrivée de Vic à Montréal et elle se termine sur son premier grand succès, au moment où il abandonne le gymnase de son oncle pour rejoindre l'écurie de Coco, un gérant mieux connu. Le parallèle entre la carrière d'Eddy, celle de Vic et celle de Marcel, dont la carrière, gérée par Eddy, est au point mort, permet de relativiser l'idée même de réussite et d'échec dans ce contexte particulier. Rien ne prouve que Vic s'en sortira mieux que les autres; mais rien n'indique pour autant que le destin sera inexorable. Seule en découle l'incontournable aliénation, doublement exprimée elle aussi, d'abord par les spectres de Jacques, qui rappellent au souvenir d'Eddy qu'il a renié sa famille (il n'a pas assisté aux funérailles de son frère) puis par le fait que Vic, pour réussir, doit lui aussi quitter son oncle Eddy.

Le choix de la boxe comme prétexte est heureux et efficace. Il inscrit la violence dans un cadre stable, à la fois réaliste (la boxe est un combat) et métaphorique (la vie est un combat). Elle agit du même coup sur la langue de la pièce, langue dure, qui cogne autant que les poings, et à laquelle s'oppose la langue de Madeleine, la femme d'Eddy, qui, si elle peut cogner autant que la langue des hommes, conserve néanmoins un certain pouvoir d'évocation, tout en demiteintes. Comme La Mort de Blanche et L'Ombre de toi, mais mieux réussie, la dernière pièce de Jean-Marc Dalpé repose elle aussi sur la fracture du sujet. L'opération sent cependant moins l'effet de rhétorique puisque la fracture du sujet est envisagée à la fois sous l'angle onirique (par les spectres) et sous un angle réaliste, le sujet francoontarien étant reconduit sur les deux vies parallèles de Vic et d'Eddy. D'autres dédoublements s'y superposent: Eddy et Coco, Maurice et 
Vic. Dalpé confirme un peu plus à chaque pièce l'immense talent dramaturgique qui lui a déjà valu le prix du Gouverneur général pour Le Chien. Eddy a fait l'objet d'une double création, en anglais d'abord au festival de Stratford, le 22 juin 1994, dans une traduction de Robert Dickson et une mise en scène de Richard Rose, puis, en français, à Montréal, le 11 octobre suivant, par la Nouvelle Compagnie théâtrale, dans une mise en scène de Brigitte Haentjens.

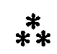

La Blanche de Chantale Boileau mourait elle aussi plusieurs fois, mais, comme l'anecdote était fondée sur la mort ultime et que la pièce effectuait une remontée dans le passé, le titre est resté au singulier, donnant ainsi une sorte d'ambiguité au sens du mot mort: mort réelle qui encadre la pièce, mais aussi mort symbolique de l'adolescente souffrant du psoriasis, de la mère perdant à la fois un fils et une fille, etc. Dans Les Quatre Morts de Marie ${ }^{6}$, créée à Louvain-la-Neuve, en Belgique, le 13 mai 1994, Carole Fréchette inverse cette perspective en proposant un personnage qui mourra quatre fois, mais dans un ordre chronologique plus conventionnel.

La pièce n'effectue donc pas de rétrospective, mais se donne comme une sorte de récit de vie, en quatre tableaux, chacun donnant lieu à une des morts de Marie. Le personnage s'en trouve saisi en quatre temps: l'enfant de douze ans qu'abandonnent ses parents; la jeune anarchiste qui veut refaire le monde en faisant sauter (littéralement) l'actuel; la jeune femme marginale qui vit dans un camion au milieu d'un terrain vague; et celle, au bout du rouleau, d'une femme encore un peu plus âgée, partie seule dans une chaloupe sur la mer. De sorte que, en entrant dans l'eau qui figure la mort réelle, Marie ne fait que clore uń processus entrepris depuis longtemps où elle était déjà morte par étapes. Entre ces quatre parties, un certain nombre de fils créent des liens: les histoires de Marie, qui réinvente son univers sur le mode de la fiction; le bruit de l'eau qui préfigure chaque rupture; les personnages secondaires, constamment les mêmes, mais sous de nouveaux noms, dans des circonstances nouvelles.

Ainsi l'action n'a pas de centre, pas de noud. Elle est organisée selon quatre temporalités distinctes, qui découpent autant de Marie différentes, réunies sous le même nom (qui est aussi un générique du féminin) et dans une même marginalité, et qui dénotent, dès le départ, une mort au monde social réel. Le résultat repose sur la lecture de la 
vie où l'enfance est le moment des grandes richesses que les épisodes ultérieurs viennent vider de leur séns peu à peu et dont la fiction n'arrive jamais à réparer les fissures. La structure des Quatre Morts de Marie rappelle ainsi celle de la première pièce de l'auteure, Baby blues, mais le discours est ici radicalement autre: à la polyphonie des mères se substituent l'immense solitude de la fille et l'échec de l'écriture à combler le manque. Depuis le temps du Théâtre des Cuisines, qui présentait la condition des femmes sous une forme épique, orientée vers la transformation du monde réel, l'écriture de Carole Fréchette n'a cessé de se replier sur elle-même, dans une vision qui, touchant l'intime de plus près, atteint ici une sorte de clôture, vision tragique d'où l'espoir est banni. Mais, comme le dit Marie, la mer est peut-être "seulement une impression" (p. 116).

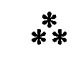

L'opéra est un genre rarement pratiqué de nos jours. Marc Gagné, qui donne ici Évangéline et $\mathrm{Gabriel}^{7}$, n'en est pas à ses premières armes, ayant déjà signé en 1987 un opéra, en trois actes et un prologue, adapté du Menaud maître-draveur de Félix-Antoine Savard. Les sujets, on le voit, renvoient au répertoire classique de la littérature québécoise, qui convient au genre pratiqué, et l'auteur met en musique des textes qui, à l'origine, tiraient déjà un important profit d'un travail sur les voix. On se rappellera les voix du Menaud, qui thématisaient la tradition et structuraient le roman. De même ici, les voix rappellent la tradition orale acadienne autant que le poème de Longfellow:

L'opéra ne permet guère d'expérimentation, du moins en ce qui concerne la théâtralité, et le genre conserve quelque chose de la tragédie classique, ici curieusement assez proche des principes moteurs du Phèdre de Ronfard: sujet connu reconstruit à partir d'un discours préexistant (le poème de Longfellow), personnages soumis aux caprices et à la mesquinerie d'un pouvoir sur lequel ils n'ont aucune prise (c'est d'abord le mythe qui intéresse Marc Gagné et non l'histoire, ce qui l'aurait obligé à envisager le point de vue britannique), effets de narration engendrés par les chours. L'opéra, en effet, comme la tragédie ou l'épopée, ne permet aucune problématisation; elle n'engage pas la réflexion, mais le chant, c'est-à-dire la célébration des personnages. Elle est également la célébration de la voix, mettant en veilleuse l'espace et le temps, au cœur de toute théâtralité. De sorte que Gagné a parfaitement raison de sous-titrer son livret "Poème drama- 
tique . On notera le déplacement du titre par rapport à l'œuvre initiale: Évangéline ici occupe un rang secondaire; Gabriel est le héros véritable de l'épopée acadienne que réécrit Marc Gagné.

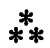

Par comparaison, En circuit fermé $e^{-8}$ de Michel Tremblay présente une structure qui est tout, sauf fragmentée. Au contraire, le sujet de la pièce est noué selon l'action classique; les personnages présentent une unité, une cohérence fermement établie; le temps est linéaire et l'espace demeure figuratif. Ce qui prime ici est la dissolution et non la fragmentation de la théâtralité. La cohérence du texte ne réside pas dans le respect des unités classiques de la dramaturgie, mais dans l'élimination de la nécessaire représentation: le texte ne laisse en effet qu'une place fort restreinte au jeu, à la spatialisation, à la mise en scène. En cela, il se rapproche davantage des dialogues platoniciens que d'une quelconque forme de théâtre. Aussi l'auteur a-t-il eu raison de baptiser son texte "Pamphlet", même si l'éditeur le présente dans sa collection "Théâtre". Par son contenu manifestaire, la pièce rappelle L'Impromptu d'Outremont, où Tremblay réglait ses comptes avec le public et les critiques bourgeois, à propos des Belles-sœurs. Il s'en distingue radicalement par sa forme, bien que le personnage principal d'En circuit fermé soit le légendaire Nelligan Beaugrand-Drapeau, fils de la Fernande de L'Impromptu, et bien que les aspirations culturelles des grandes bourgeoises d'Outremont y soient encore une fois représentées par le comité d'évaluation des programmes présidé par Sonia Bergevin.

Nommé à la direction de la télévision d'État, Nelligan BeaugrandDrapeau entend rétablir la vocation culturelle (au sens de Grande Culture) du réseau. Devant lui, se trouve le directeur sortant, Gaston Bergevin, qui avait fait d'une culture populiste (et non populaire) fondée sur l'humour "gras" l'essentiel de sa programmation. Il en résulte une satire du fonctionnarisme et de la politique, déjà inscrite dans l'œuvre de Tremblay depuis Le Gars de Québec, traduction et adaptation du Revizor de Gogol, mais ici dirigée en particulier contre les pharaons de la culture. S'y trouvent dénoncées la logique de la décision par sondage, qui révèle une absence totale de projet politique au profit d'une structure décisionnelle fluctuant selon les cotes d'écoute; l'opportunisme de la presse, représentée ici par Amanda Gariépy, gourmande et mesquine, qui sous des pseudonymes divers monopolise tout l'espace critique de la ville et fait chanter la 
direction de la télévision à sa guise; et les pratiques de collusion que mènent la chroniqueuse Sybille Berger, laquelle doit sa carrière non à son talent (qui n'est pas grand), mais à sa dextérité sexuelle, et Robert "Bob" Beaulieu, maître de la production privée, deux fois subventionnée, d'abord par Télé-Film Canada qui finance la production, puis par la télévision d'État qui achète le produit.

Aucun personnage ne survit à la plume satirique de Tremblay, et sa critique des politiques culturelles sonne juste à un moment où la valse des directeurs, aux deux télévisions d'État que sont RadioCanada et Radio-Québec, met mieux en évidence les aspects contradictoires et parfois ridicules des choix gouvernementaux en matière de culture. On a ainsi tort de lire En circuit fermé comme une pièce à clé, dont il faudrait identifier nominalement les actants, et comme les commentaires nés de la lecture publique qu'a dirigée René Richard Cyr, au Théâtre du Nouveau Monde, le 9 mai 1994, ne s'étaient pas gênés pour le faire. Ce qu'il faut plutôt chercher ici, c'est l'acte politique d'un dramaturge dont toute l'œuvre appelle une troisième voie, entre la Grande Culture figée et le populisme toujours un peu épais, et qui se trouve évincé de l'espace culturel géré par les pouvoirs publics, marginalisé par des politiques incohérentes et qui, profitant de son importance dans le champ de la culture, cherche à faire entendre la voix des artistes. Là est l'essence même du pamphlet. Et l'on doit admettre que la plume de Tremblay, bavarde, méchante et incisive comme aucune autre, excelle dans cet art soudainement redevenu au goût du jour.

1. Jean-Pierre Ronfard, Cinq Études, Montréal, Leméac, coll. «Théâtre *, 1994, 134 p.

2. On lira à ce propos les commentaires de Ronfard dans Robert Lévesque, Entretiens avec Jean-Pierre Ronfard suivis de.La Leçon de musique 1644, Montréal, Liber, coll. 'De vive voix", 1993, 176 p.

3. Chantale Boileau, Les Formes du tragique. Essai suivi de La Mort de Blanche. Théâtre, Montréal, VLB éditeur, 1994, 188 p.

4. Sylvie Provost, L'Ombre de toi. Théâtre, Montréal, VLB éditeur, 1994, 123 p.

5. Jean-Marc Dalpé, Eddy. Pièce en cinq actes, postface de Robert Claing, Montréal, Boréal, et Sudbury, Prise de parole, coll. "Théâtre ", 1994, 203 p.

6. Carole Fréchette, Les Quatre Morts de Marie. Théâtre, Montréal, Les Herbes rouges, $1995,116 \mathrm{p}$.

7. Marc Gagné, Évangéline et Gabriel. Poème dramatique. Livret d'opéra en deux actes partiellement inspiré du poème Évangéline de H.-W. Longfellow, musique de l'auteur, œuvres de Richard Durand, Québec, Le Loup de Gouttière, 1994, 129 p.

8. Michel Tremblay, En circuit fermé, préface de Pierre Filion, Montréal, Leméac, coll. «Théâtre», 1994, 124 p. 